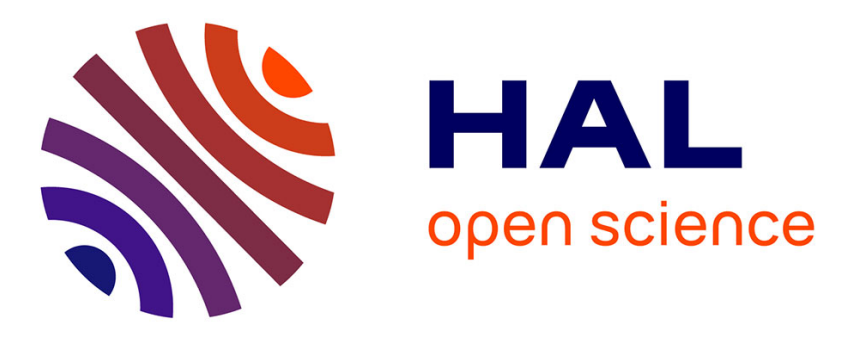

\title{
Structural Basis for Natural Product Selection and Export by Bacterial ABC Transporters
}

\author{
Maria Romano, Giuliana Fusco, Hassanul G Choudhury, Shahid Mehmood, \\ Carol V Robinson, Séverine Zirah, Julian D Hegemann, Ewen Lescop, \\ Mohamed A Marahiel, Sylvie Rebuffat, et al.
}

\section{To cite this version:}

Maria Romano, Giuliana Fusco, Hassanul G Choudhury, Shahid Mehmood, Carol V Robinson, et al.. Structural Basis for Natural Product Selection and Export by Bacterial ABC Transporters. ACS Chemical Biology, 2018, 13 (6), pp.1598 - 1609. 10.1021/acschembio.8b00226 . hal-03301757

\section{HAL Id: hal-03301757 https://hal.science/hal-03301757}

Submitted on 27 Jul 2021

HAL is a multi-disciplinary open access archive for the deposit and dissemination of scientific research documents, whether they are published or not. The documents may come from teaching and research institutions in France or abroad, or from public or private research centers.
L'archive ouverte pluridisciplinaire HAL, est destinée au dépôt et à la diffusion de documents scientifiques de niveau recherche, publiés ou non, émanant des établissements d'enseignement et de recherche français ou étrangers, des laboratoires publics ou privés. 


\title{
Structural Basis for Natural Product Selection and Export by Bacterial ABC Transporters
}

\author{
Maria Romano, ${ }^{\dagger \neq}, \bigcirc$ Giuliana Fusco, ${ }^{\dagger, \bigcirc}$ Hassanul G. Choudhury, ${ }^{\dagger \dagger}$ Shahid Mehmood, ${ }^{\S}$ \\ Carol V. Robinson, ${ }^{\S}$ Séverine Zirah, $\|$ Julian D. Hegemann, ${ }^{\perp, \#, \triangleleft}$ Ewen Lescop, ${ }^{\nabla}$ Mohamed A. Marahiel, ${ }^{\perp}$ \\ Sylvie Rebuffat, $"$ Alfonso De Simone, ${ }^{\dagger}$ and Konstantinos Beis ${ }^{*}, \dagger,+\infty$ \\ ${ }^{\dagger}$ Department of Life Sciences, Imperial College London, South Kensington, London SW7 2AZ, United Kingdom \\ ${ }^{\ddagger}$ Rutherford Appleton Laboratory, Research Complex at Harwell, Oxfordshire OX11 0DE, United Kingdom \\ ${ }^{\S}$ Department of Chemistry, University of Oxford, South Parks Road, Oxford OX1 3QZ, United Kingdom \\ "Communication Molecules and Adaptation of Microorganisms Laboratory (MCAM, UMR 7245 CNRS-MNHN), Sorbonne \\ Universités, Muséum National d'Histoire Naturelle, Centre National de la Recherche Scientifique, CP 54, 57 rue Cuvier 75005 Paris, \\ France \\ ${ }^{\perp}$ Department of Chemistry/Biochemistry, LOEWE Center for Synthetic Microbiology, Philipps-Universität Marburg, \\ Hans-Meerwein-Strasse 4, 35032 Marburg, Germany \\ "Roger Adams Laboratory, Department of Chemistry, University of Illinois at Urbana-Champaign, 600 S. Mathews Avenue, Urbana, \\ Illinois 61801, United States \\ ${ }^{\nabla}$ Institut de Chimie des Substances Naturelles, CNRS UPR 2301, Université Paris-Sud, Université Paris-Saclay, 1 av. de la Terrasse, \\ 91198 Gif-sur-Yvette, France
}

\section{Supporting Information}

ABSTRACT: Bacteria under stress produce ribosomally synthesized and post-translationally modified peptides (RiPPs) to target closely related species, such as the lasso peptide microcin J25 (MccJ25). These peptides are also toxic to the producing organisms that utilize dedicated $A B C$ transporters to achieve self-immunity. MccJ25 is exported by the Escherichia coli $\mathrm{ABC}$ transporter McjD through a complex mechanism of recognition that has remained elusive. Here, we used biomolecular NMR to study this interaction and identified a region of the toxic peptide that is crucial to its recognition by the $A B C$ transporter. Our study provides
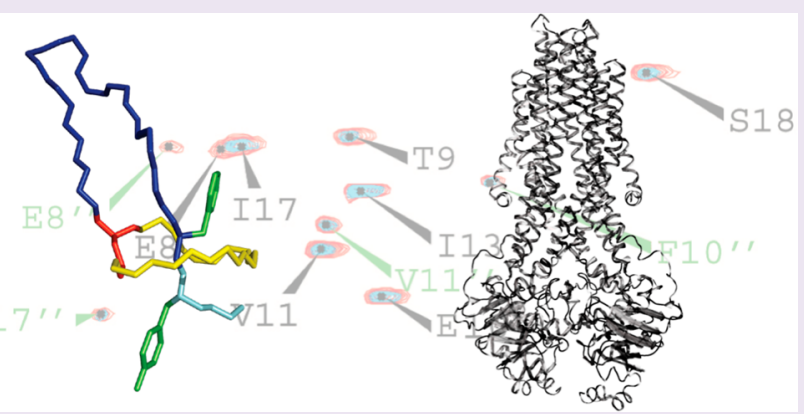
evidence that McjD is highly specific to MccJ25 and not to other RiPPs or antibiotics, unlike multidrug ABC transporters. Additionally, we show that MccJ25 is not exported by another natural product $A B C$ transporter. Therefore, we propose that specific interactions between natural product $A B C$ transporters and their substrate provides them with their high degree of specificity. Taken together, these findings suggest that $A B C$ transporters might have acquired structural elements in their binding cavity to recognize and allow promiscuous export of a larger variety of compounds.

$\mathrm{T}^{\mathrm{k}}$ he increase in the resistance to antibiotics has become a major challenge in the treatment of bacterial infections, particularly those caused by multidrug resistant bacteria, and finding novel molecules and strategies to fight these super bugs is of critical urgency. The therapeutic potential of natural products is generally recognized as a promising route to develop valuable drug leads. ${ }^{1}$ Microbial metabolites, including antimicrobial peptides and peptide-derived natural products, are the major source of antimicrobials currently used in human and animal health, food safety, and agriculture. Peptide natural products mainly arise from two biosynthesis pathways, ${ }^{2}$ which generate chemical diversity that is reflected in their interactions with various cellular targets and hence their biological properties.
These biosynthesis pathways either use large modular enzymatic complexes, the nonribosomal peptide synthetases (NRPSs), ${ }^{3}$ or are produced via the ribosomal peptide synthesis followed by extensive post-translational modifications of the protein precursors by various dedicated enzymes to produce the mature compounds. ${ }^{4}$ The huge structural diversity of the resulting socalled ribosomally synthesized and post-translationally modified peptides (RiPPs) has only recently emerged, and the number of known families of RiPPs has expanded considerably in recent

Received: March 8, 2018

Accepted: May 14, 2018

Published: May 14, 2018 
years. RiPP families have been defined on the basis of the primary post-translational modifications, ${ }^{5}$ although many RiPPs are further modified by enzymes. ${ }^{6}$ Generally, the precursor peptide contains an $\mathrm{N}$-terminal leader sequence, which is involved for a part in recognition by the modification enzymes and is cleaved off by specific proteases, and a C-terminal core sequence, which harbors the post-translational modification sites and becomes the active entity. ${ }^{5}$ Although the complex mechanisms involved in the biosynthesis of RiPPs are continuously studied, the way they are exported out of the producing cells using export machineries, such as $\mathrm{ABC}$ transporters, is poorly documented. Moreover, this export process is often tightly linked to self-immunity of the producer strain to its own toxic compound, making it resistant to the produced RiPP. ${ }^{5}$ Deciphering these export mechanisms thus remains a challenge of crucial importance to advance the field.

MccJ25, an antimicrobial RiPP produced by Escherichia coli under nutrient exhaustion to contribute to microbial competitions, is the archetype of lasso peptides, which are among the most extensively studied RiPPs. ${ }^{4,5}$ The lasso topology consists of an $\mathrm{N}$-terminal macrolactam ring where the $\mathrm{C}$-terminal tail is threaded and sterically trapped, forming an antiparallel $\beta$-hairpin loop (Figure 1a,b). ${ }^{7}$ MccJ25 is synthesized by the $\mathrm{McjB} / \mathrm{C}$ protein complex, which ensures establishment of the lasso topology from the McjA precursor. ${ }^{8}$ MccJ25 exerts potent

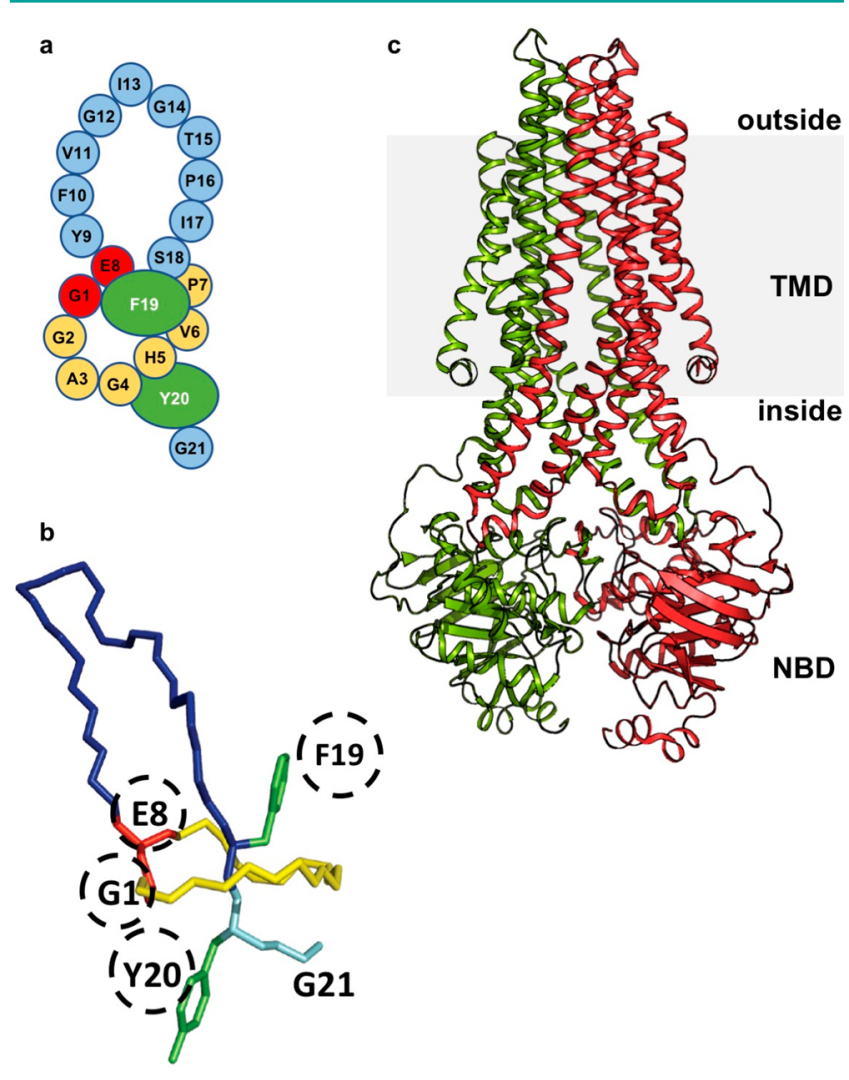

Figure 1. Structures of Mccj25 and McjD. (a) Schematic of the lasso topology of MccJ25. Its amino acid sequence is shown in a ball model. (b) Three-dimensional NMR structure of MccJ25 (PDB 1Q71). ${ }^{7}$ The macrolactam ring, the isopeptide bond between the G1 amino group and the E8 side chain carboxylate, the Y9-S18 loop region, and the two bulky aromatic residues F19 and Y20 serving as a lock for maintaining the lasso topology are in yellow, red, blue, and green, respectively (as in panel a). (c) Crystal structure of apo-McjD (PDB 5OFP). ${ }^{15}$ The two monomers are shown as green and red cartoons, and the membrane is depicted as a gray box. antibacterial activity against Escherichia and Salmonella species through RNA polymerase inhibition. ${ }^{9}$ In E. coli, MccJ25 hijacks the outer membrane siderophore receptor FhuA/TonB-ExbBExbD dependent pathway for uptake in the target bacteria ${ }^{10}$ and the inner membrane SbmA protein transporter. ${ }^{11,12}$ Our group has previously studied the structure and function of FhuA in complex with MccJ25, which sheds light on the hijacking mechanism. ${ }^{13}$ Export of MccJ25 out of the producer cells permits both exerting antibacterial activity for competition against other bacteria occupying the same niche and providing the producer with self-protection against the antibacterial lasso peptide. In $E$. coli, these functions are performed by the $\mathrm{ABC}$ transporter $\mathrm{McjD}^{14,15}$ (Figure 1c).

Currently, the only functionally and structurally characterized peptide-derived natural product $\mathrm{ABC}$ transporter is $\mathrm{McjD}$. ${ }^{14,15,16}$ We have previously determined the crystal structure of $\mathrm{McjD}$ in multiple conformations that has allowed us to propose a detailed mechanism for MccJ25 export from producing cells. ${ }^{14,15}$ The core architecture of $\mathrm{McjD}$ is composed of a dimeric transmembrane domain (TMD) of 12 transmembrane (TM) helices, which forms the translocation pathway across the membrane bilayer and contains the ligand binding site, and a dimeric nucleotide binding domain (NBD) where ATP binds and is hydrolyzed (Figure 1c). McjD uses the alternating access mechanism switch between inward- and outward-facing states, which exposes the ligand binding site alternatively to the inside or outside of the membrane, coupled to ATP binding and hydrolysis. However, unlike multidrug exporters for which the TMD is either open to the cytoplasmic or periplasmic space, the TMD of McjD is mostly occluded. Our functional data have shown that the cavity opens transiently to allow export of MccJ25. We have proposed that the occluded cavity probably provides specificity to the produced peptide. However, despite the availability of several structures of $\mathrm{ABC}$ transporters, most have been determined in the absence of their ligand. ${ }^{17}$ To date, the only structural information on a bacterial $A B C$ exporter associated with its substrate is from the crystal structure of the bacterial NaAtm 1 in complex with glutathione derivatives ${ }^{18}$ and the cryo-EM structure of MsbA with lipid A. ${ }^{19}$

In this study, we sought to investigate the recognition mechanism of MccJ25 by $\mathrm{McjD}$ reconstituted in bicelles (membrane mimetic) using solution nuclear magnetic resonance (NMR). Our data revealed that 4 out of 21 amino acids composing mature MccJ25 are involved in binding and recognition by $\mathrm{McjD}$, a result that is also supported by our ligand induced ATPase data in proteoliposomes. We also probed the specificity of $\mathrm{McjD}$ for MccJ25 by measuring its ligand induced ATPase activity in the presence of different posttranslationally modified peptides and multidrug $\mathrm{ABC}$ transporter substrates in proteoliposomes. Interestingly, only MccJ25 is capable of inducing the ATPase activity, suggesting that McjD is highly specific for its native substrate. Additionally, our cytotoxicity assays show that $\mathrm{McjD}$ cannot confer resistance to various drugs when overexpressed in drug hypersensitive $E$. coli cells. We also show that another lasso peptide $A B C$ transporter, capistruin $\mathrm{ABC}$ transporter CapD from Burkholderia, cannot provide immunity to $E$. coli cells in the presence of MccJ25. Altogether, these data provide evidence that the $\mathrm{ABC}$ transporters involved in export of RiPPs and bacterial self-immunity are very specific toward their substrates. 
a
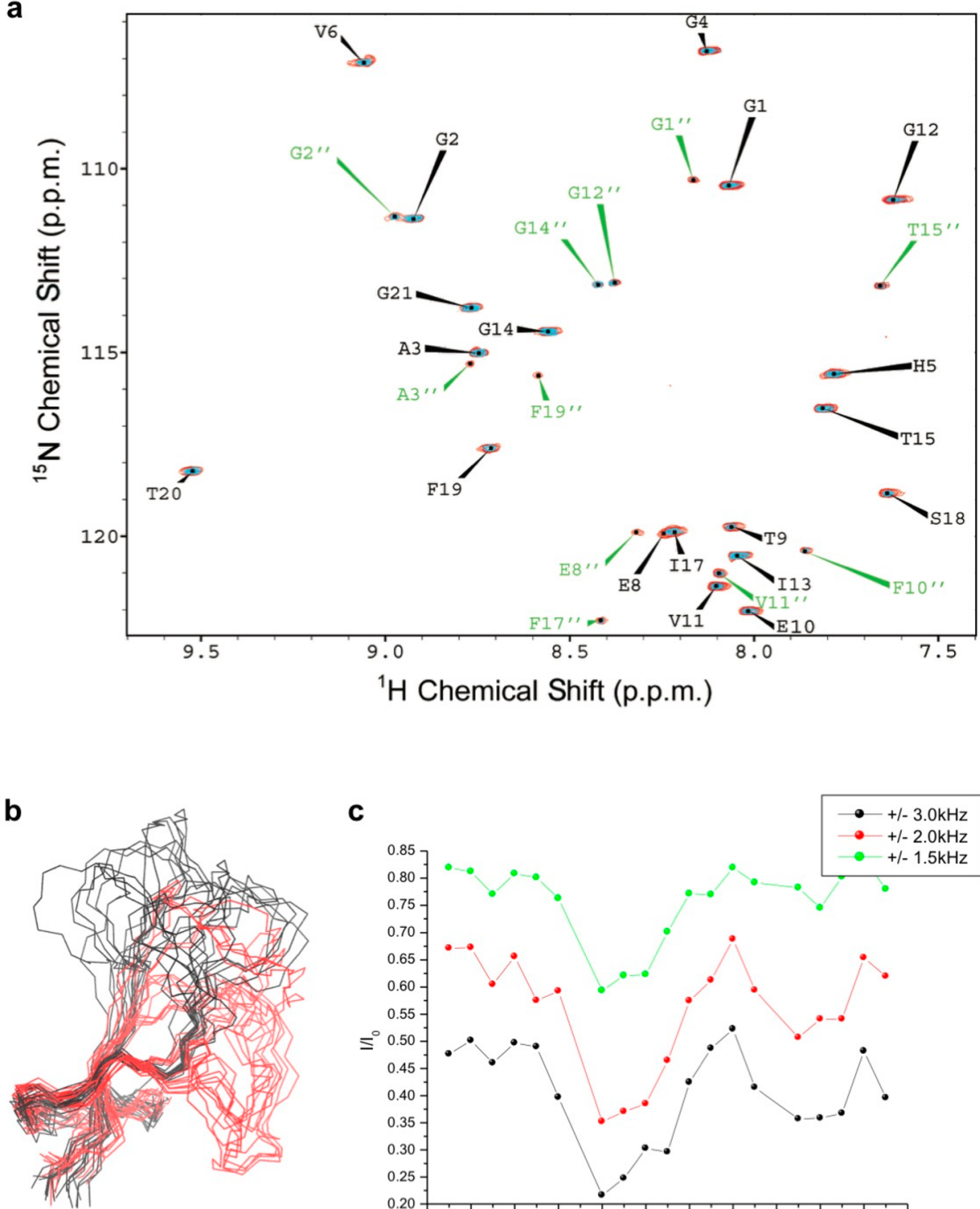

C

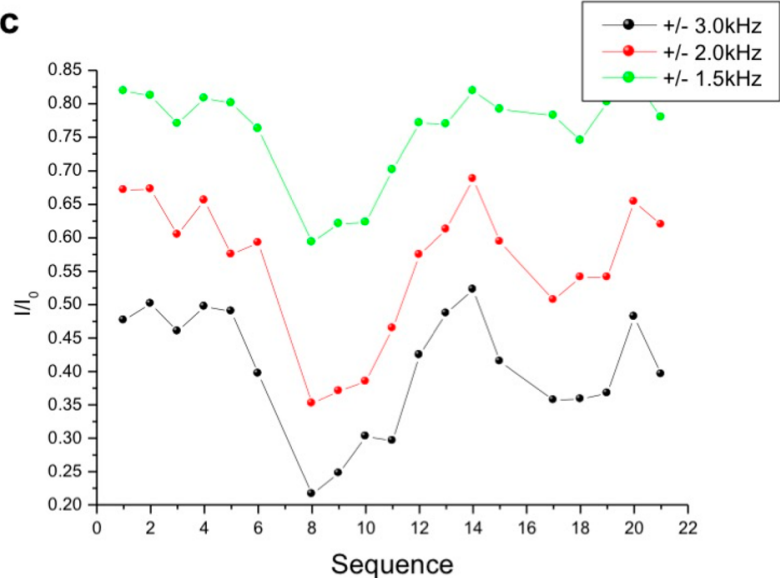

d
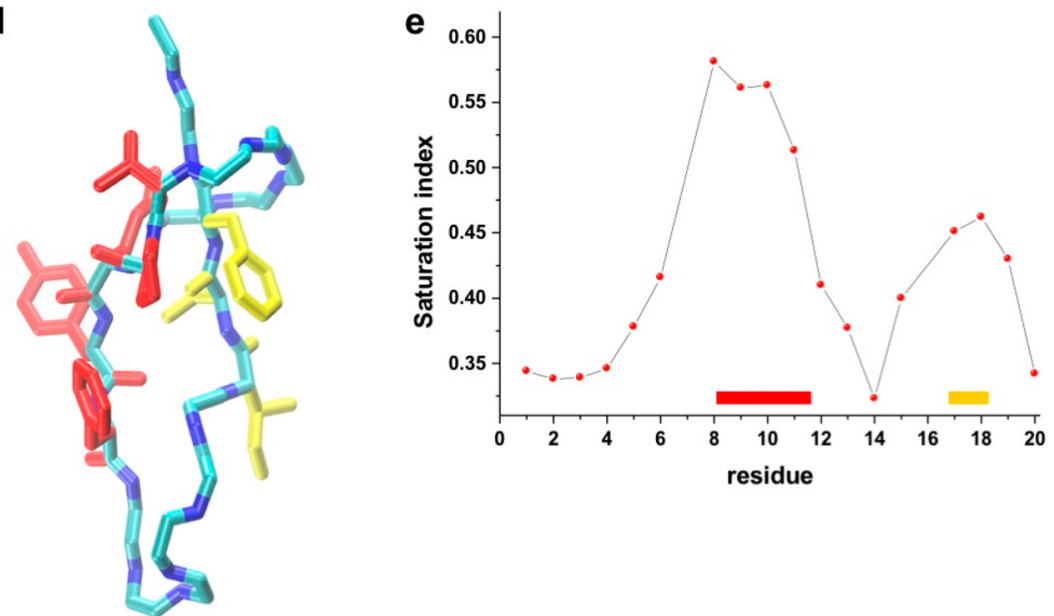

Figure 2. NMR analysis of the MccJ25-McjD interaction. (a) CEST ${ }^{1} \mathrm{H}-{ }^{15} \mathrm{~N}$ HSQC experiments of MccJ25 in phosphate buffer measured at $310 \mathrm{~K}$ using an $800 \mathrm{MHz}$ instrument with a cryoprobe TCI. Cyan and red spectra report the CEST measured with an offset of $1.5 \mathrm{kHz}$ and $100 \mathrm{kHz}$, respectively. Individual CEST profiles are shown in Supporting Information Figure 3 and control experiments with MccJ25 and bicelles are shown in Supporting Information Figure 6. (b) Chemical-shift refined structures of MccJ25 in its main primary (black) and secondary (red) conformations 
Figure 2. continued

amounting to $90 \%$ and $10 \%$ of the solution state of the peptide at $310 \mathrm{~K}$. (c) CEST profiles of MccJ25 in the presence of McjD measured at different saturation frequencies and shown as a function of the residue number. The plot reports the average values of CEST saturations measured using offsets of $+1.5 \mathrm{kHz}$ and $-1.5 \mathrm{kHz}$ (black), $+2.0 \mathrm{kHz}$ and $-2.0 \mathrm{kHz}$ (red), and $+3.0 \mathrm{kHz}$ and $-3.0 \mathrm{kHz}$ (green). (d) Primary (red residues) and secondary (yellow residues) regions in MccJ25 showing high level of CEST saturation upon interaction with McjD. (e) Saturation index (see Methods) derived from the analysis of the CEST spectra, indicating the primary (marked in red) and secondary (marked in yellow) regions experiencing high saturation transfer in MccJ25.

\section{RESULTS AND DISCUSSION}

McjD-MccJ25 Interaction by NMR. Using biomolecular NMR, we investigated the propensity of MccJ25 to interact with $\mathrm{McjD}$ and its conformational properties in solution. The conformations adopted by ${ }^{15} \mathrm{~N}-\mathrm{Mcc} / 25$ in water appeared to be different from those found in methanol. ${ }^{7}$ In particular, we found that the peptide adopts two conformational states that are detected in slow exchange in the ${ }^{1} \mathrm{H}-{ }^{15} \mathrm{~N}-\mathrm{HSQC}$ spectrum, leading to dual resonances for residues G1, G2, and E8 in the macrolactam ring; residues F10, V11, G12, G14, G15, and F17 in the loop; and residue F19 (Figure 2a and Supporting Information Figure 1). The largest chemical shift differences are observed for residues F10 to F19 that define the loop region and its C-terminus (Supporting Information Figure 2), indicating that the conformational differences between the two forms are mostly located in the loop region. The average ratio of the peak intensities between the dual conformations of these residues indicate that at $310 \mathrm{~K}$ the secondary state has a population of $10 \% .{ }^{13} \mathrm{C}$ resonances from $\mathrm{HNCO}$ and CBCAcoNH spectra (see Methods) indicated two additional residues in conformational exchange, namely, P7 and P16. Notably, the secondary state of P16 adopts a cis conformation when the peptide is in its secondary state (Supporting Information Table 1), as indicated by a $2.1 \mathrm{ppm}$ downfield shift for the ${ }^{13} \mathrm{C} \beta$ and a $0.6 \mathrm{ppm}$ upfield shift for the ${ }^{13} \mathrm{C}$ carbonyl resonances. The cis/trans isomerization of P16 is likely to be at the origin of the slow conformational exchange between the two structural states of MccJ25 (Figure 2a). We also found that the exchange rate for the interconverting states is at the second time scale at $318 \mathrm{~K}$, which is in agreement with cis/trans proline isomerization (Supporting Information Figure 1). The chemical shift of the backbone atoms of the two states of MccJ25 enabled us to refine their conformational ensembles ${ }^{20}$ (Figure $2 b$ ). The ensemble indicates that the two conformational states of MccJ25 adopt two significantly different overall orientations of the bundles of the major loop of the lasso peptide, likely as a result of the different backbone conformations of P16 in its cis and trans forms.

We then employed the NMR experiments of chemical exchange saturation transfer (CEST), ${ }^{21,22,23,24,25,26,27}$ to directly probe the nature of the interaction between Mccj25 and the $A B C$ transporter McjD. CEST is very sensitive to the equilibrium between low molecular weight species (observable with solution NMR) and transiently bound states with slow-tumbling highmolecular-weight complexes, which indeed cannot be observed directly in solution NMR as a result of excessive line broadening. ${ }^{21,22,23,24,25}$ In our implementation of CEST, we used a continuous weak radiofrequency band of $500 \mathrm{~Hz}$, applied off-resonance (by up to $\pm 9 \mathrm{kHz}$ ) in the ${ }^{15} \mathrm{~N}$ channel, to saturate the broad spectroscopic transitions in the McjD-bound state of MccJ25 that remained NMR undetectable because it has an overall mass of $\sim 250 \mathrm{kDa}$, including the bicelles, but leaving the resonances of the free MccJ25 state (NMR detectable) virtually unperturbed. Saturation of the bound state is then transferred to the free state via exchange between bound and unbound states of MccJ25, attenuating the intensities of the observable resonances of the latter. We carried out CEST experiments with $100 \mu \mathrm{M}$ of MccJ25 in the presence of $8 \mu \mathrm{M}$ of McjD embedded in bicelles. At this MccJ25 to McjD concentration ratio, the ${ }^{1} \mathrm{H}-{ }^{15} \mathrm{~N}-\mathrm{HSQC}$ resonances exhibited minimal resonance broadening, which is a convenient setup to maximize the sensitivity of CEST. The CEST profiles (Figures $2 \mathrm{c}$ and Supporting Information Figure 3) identified a group of residues featuring significant levels of saturation transfer, indicating that they are primarily involved in the interaction with McjD. These residues are clustered in two main regions of MccJ25, with the 8-11 segment EYFV (Figure $2 \mathrm{~d}$ ) being associated with the strongest levels of saturation transfer, indicating that this region is the primary site of interaction with McjD (Figure 2e). Additional intermediate levels of CEST saturation were found in the region 17-19 of sequence ISF. The intermediate saturation associated with region 17-19 can be explained by both a direct, but weaker, interaction with the $A B C$ transporter or with an induced saturation transfer due to the strong packing with the primary binding region, 8-11 (Figure 2d).

The analysis of the CEST profiles associated with the second conformation of Mccj25 provided similar indications to those obtained with the main conformational state (Supporting Information Figures 4 and 5), suggesting that the conformational exchange does not influence significantly the binding properties of the lasso peptide. This finding is consistent with the limited structural diversity within regions $8-11$ and $17-19$ in the two conformational states of Mccj25 (Figure 2b).

Characterization of the Interaction of McjD with an MccJ25 Variant. The export efficiency was evaluated for MccJ25 and a deletion variant lacking F10 and V11, namely, MccJ25- $\Delta \mathrm{FV}$, which however retains the lasso structure; ${ }^{28,29}$ measurements of the relative quantity of peptide detected in pellets and supernatants by LC-MS indicated a weak decrease in the transport efficiency for MccJ25- $\Delta \mathrm{FV}^{28}$ To gain further insights into the mechanism of recognition, binding, and export, we used the MccJ25- $\Delta \mathrm{FV}$ variant (Figure $3 \mathrm{a}$ ) to perform ligand induced ATPase assays with $\mathrm{McjD}$ reconstituted in liposomes. McjD displayed a basal ATPase activity of $49.7 \mathrm{nmol} \mathrm{min}{ }^{-1} \mathrm{mg}^{-1}$ protein that was increased to $73.7 \mathrm{nmol} \mathrm{min}{ }^{-1} \mathrm{mg}^{-1}$ upon stimulation by Mccj25 (Figure 3b). These values are in agreement with our previous results where the basal ATPase activity of $44.4 \pm 0.5 \mathrm{nmol} \mathrm{min}{ }^{-1} \mathrm{mg}^{-1}$ protein was stimulated in a concentration-dependent manner. ${ }^{14}$ By contrast, Mccj25- $\Delta \mathrm{FV}$ did not stimulate the ATPase activity of McjD, suggesting that the F10-V11 residues in the loop region are essential for the recognition and binding to $\mathrm{Mcj} \mathrm{D}$, further validating our NMR model.

Since MccJ25- $\Delta \mathrm{FV}$ is exported with lower efficiency compared to MccJ25, ${ }^{28}$ we further characterized its interaction with $\mathrm{McjD}$ by nondenaturing mass spectrometry (MS). Nondenaturing MS has shown that it preserves membrane protein interaction with ligands and drugs. ${ }^{30}$ Since we have 

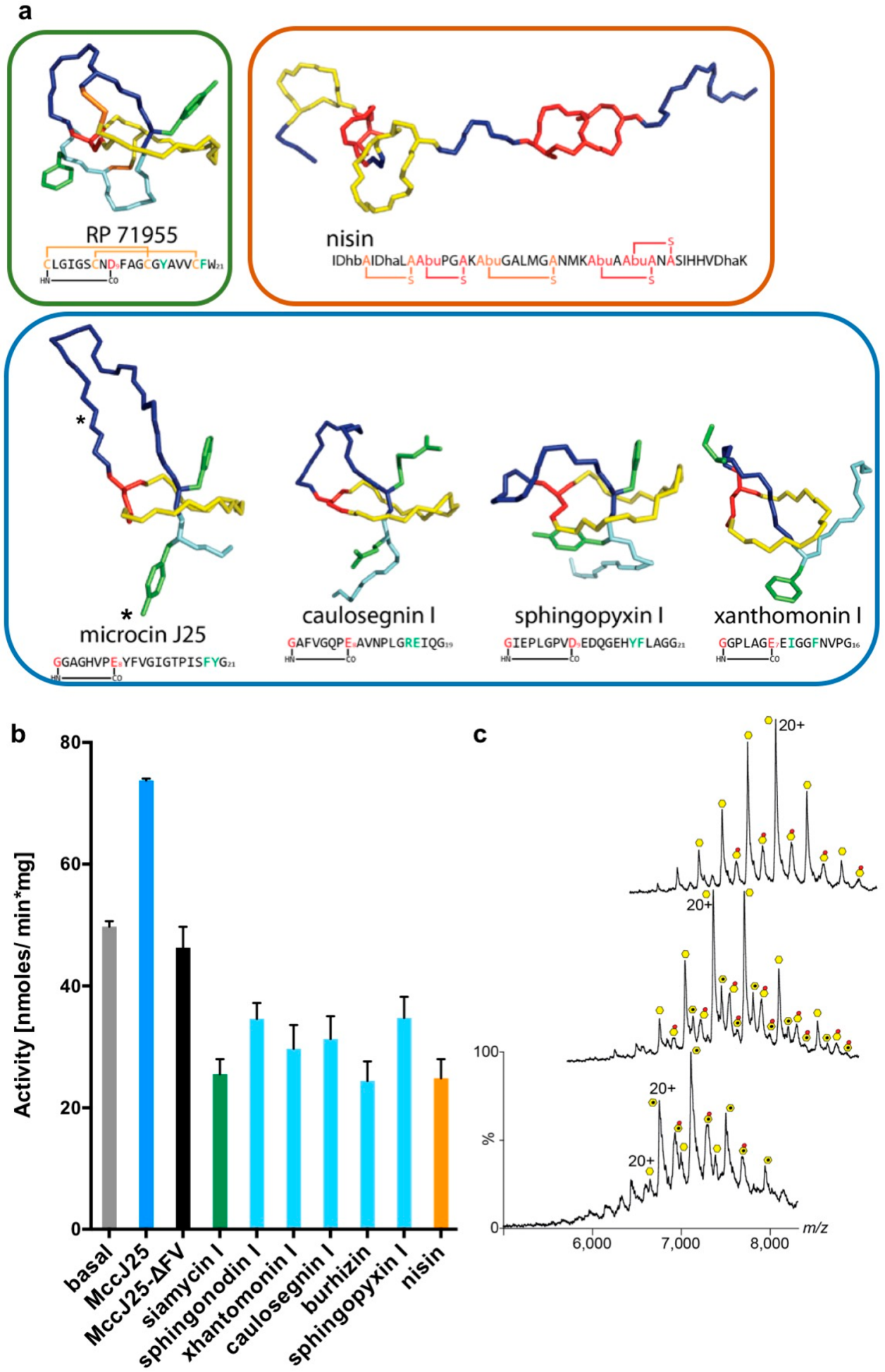

Figure 3. Functional characterization of McjD in the presence of different RiPPs. (a) Overview of all available 3D structures of peptides used in this study. The lasso peptides (MccJ25 (PDB code 1Q71), RP 71955 (PDB code 1RPB), caulosegnin I (PDB code 2LX6), sphingopyxin I (PDB code 5JQF), xanthomonin I (PDB code 4NAG)) are colored coded as in Figure 1. Disulfide bonds are in orange. The lantipeptide nisin (PDB code 1WCO) is color coded by residues outside of the (methyl)lanthionone rings (blue) and the rings itself (alternating yellow and red); Dha = dehydroalanine, Dhb = dehydrobutyrine, $\mathrm{Abu}=$ aminobutyrine. $\mathrm{RP} 71955$ was used to represent the closely related siamycin I structure (the only differences in primary structures are found at position 4 (Val for siamycin I, Ile for RP71955) and position 17 (Ile for siamycin I, Val for RP71955)). Each RiPP structure is boxed in different color; green for class I, blue for class II, and orange for the lantipeptide. (b) The basal ATPase activity of McjD is induced by MccJ25 but not by other RiPPs. The bars are color coded to distinguish between the different RiPPs classes as in panel (a). Error bars are shown for all measurements (mean \pm SEM; $n=3$ ). (c) Mass spectrum of McjD reveals two series of charge state peaks consistent with apo McjD and LPS bound adduct with $20+$ main charge state (top panel). Addition of MccJ25- $\Delta \mathrm{FV}$ (middle panel) and MccJ25 (bottom panel) at a final concentration of $8 \mu \mathrm{M}$ to $\mathrm{McjD}$ leads to the formation of a complex with protein and LPS bound peaks. A much higher degree of complex formation is observed for MccJ25 than Mccj25- $\Delta$ FV. Deconvoluted masses of apo, LPS, and peptide bound peaks are in agreement with theoretical masses. Apo McjD, LPS bound McjD, and 
Figure 3. continued

peptide (MccJ25 or MccJ25- $\Delta \mathrm{FV}$ ) bound McjD peaks are labeled with a yellow hexagon, a small black circle inside hexagon, and a small circle outside the hexagon, respectively. The population of McjD bound to both LPS and peptide is labeled as a hexagon with a black circle inside and a small red circle outside the hexagon.

previously shown that the interaction of McjD with MccJ25 is preserved in the gas phase of native mass spectrometry, ${ }^{16}$ we applied the method to probe the interaction between McjD and the MccJ25- $\Delta \mathrm{FV}$ variant. As previously shown, a clear charge state resolved mass spectrum of $\mathrm{McjD}$ with adduct peaks of LPS was obtained under high activation conditions, ${ }^{16}$ and binding of MccJ25 to McjD was preserved. Nondenaturing mass spectrometry allowed us to compare the affinities of $\mathrm{McjD}$ for $\mathrm{MccJ} 25$ and MccJ25- $\Delta$ FV. Mass spectra at different concentrations of MccJ25 showed an increase in the peptide adduct peak for all charge states (Supporting Information Figure 7). The mass

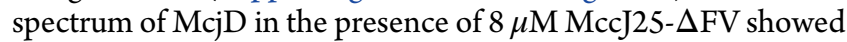
three series of charge states corresponding to apo-McjD, the LPS adduct, and a new peak with $\mathrm{m} / z$ value intermediary between those for the apoprotein and the LPS adduct (Figure 3c). Deconvolution of the new charge states provided a mass consistent with that of MccJ25- $\Delta \mathrm{FV}$. Therefore, the data suggest that $\mathrm{McjD}$ weakly interacts with Mccj25- $\Delta \mathrm{FV}$ under our experimental conditions. In the presence of MccJ25- $\Delta \mathrm{FV}$, we did not find any adduct peaks below $8 \mu \mathrm{M}$ concentration, and due to solubility issues under nondenaturing MS conditions, we were unable to achieve higher peptide concentrations. Therefore, these results clearly indicate that the $\mathrm{ABC}$ transporter $\mathrm{McjD}$ has less affinity for MccJ25- $\Delta \mathrm{FV}$ than for MccJ25.

McjD Recognizes and Transports MccJ25 Exclusively. Both bacterial and eukaryotic $\mathrm{ABC}$ exporters are implicated in multidrug resistance, as a result of multisubstrate recognition and transport. We have previously proposed that shielding of the McjD cavity might provide specificity for MccJ25 and not for other substrates unlike multidrug $\mathrm{ABC}$ transporters. As the ligand induced ATPase activity of the eukaryotic multidrug $A B C$ transporter P-glycoprotein ${ }^{31}$ and the bacterial lipid A ABC transporter $\mathrm{MsbA}^{32}$ reconstituted in proteoliposomes can be significantly stimulated in the presence of various drugs, we sought to investigate the specificity of $\mathrm{McjD}$ for other posttranslationally modified peptides and antibiotics using ligand induced ATPase assays in proteoliposomes. In particular, lasso peptides can be classified in three classes depending on the mode of stabilization of the [1] rotaxane structure that subtends the lasso topology: ${ }^{33,34,35}$ classes I and III include peptides stabilized by two and one disulfide bridge aided by steric interactions, respectively, and class II, which includes MccJ25, assembles peptides devoid of disulfide bridges and where strong steric hindrance from bulky residue side chains tightly locks the lasso fold. Among these three classes, we have selected siamycin $\mathrm{I}^{36}$ (class I), burhizin ${ }^{36} /$ caulosegnin $\mathrm{I}^{37} /$ sphingopyxin $\mathrm{I}^{38} /$ sphingonodin $\mathrm{I}^{36}$ and xanthomonin $\mathrm{I}^{39}$ (class II with different sizes of loops and rings), and BI-32169 ${ }^{36}$ (class III; Figure 3a), since we could produce and purify them in sufficient quantities, and also included a RiPP from Lactococus lactis, the lantipeptide nisin (Figure 3a), currently used as a food preservative, ${ }^{40}$ and commercial antibiotics or anticancer drugs (oxacillin, novobiocin, verapamil, doxorubicin). The aforementioned post-translationally modified peptides require an $\mathrm{ABC}$ transporter for export and self-immunity at the end of their biosynthetic cluster (with the exception of caulosegnin I, sphingopyxin I, sphingonodin I, and xanthomonin I). Interestingly, none of the peptides selected in this study was capable of stimulating the ATPase activity of $\mathrm{McjD}$ (Figure $3 \mathrm{~b}$ ), including lasso peptides of the same class II as Mcc525, and a small reduction in the ATPase activity was observed for all the peptides. The BI-32169 peptide precipitated under our assay conditions and was excluded from the analysis. In contrast, the antibiotic oxacillin and the anticancer drug verapamil showed strong inhibition of the ATPase activity, while the antibiotic novobiocin and the cancer drug doxorubicin had no effect on the ATPase activity of McjD (Figure 4a).

In addition to the ATPase activity assays, we further investigated if any of these drugs are actively exported by $\mathrm{McjD}$ in vivo by measuring their cytotoxic effect on drug hypersensitive E. coli cells, BL21 (DE3) $\triangle$ acrAB, expressing $\mathrm{McjD}$. The RiPPs were excluded from the in vivo cytotoxicity assays as they do not have antibacterial activity against $E$. coli. $^{36,37,38,39,40}$ The toxicity measurements revealed that McjD is not capable of providing the cells with immunity against the set of drugs here employed, further confirming that it is not a multidrug $\mathrm{ABC}$ transporter (Figure $4 \mathrm{~b}-\mathrm{e}$ ). Cells treated with verapamil showed delayed growth that could be due to slow active transport by $\mathrm{McjD}$, which could also be explained by the significantly reduced ATPase activity of $\mathrm{McjD}$ in its presence. Using the ATP hydrolysis deficient mutant E506Q, the cells are still capable of showing delayed growth, suggesting that $\mathrm{McjD}$ is not involved in verapamil transport (Figure 4e). The cell growth would probably be a result of another resistance mechanism that would be McjDindependent. While the cytotoxicity data suggest that all the drugs here tested are not transported by $\mathrm{McjD}$, the ligand stimulated ATPase data indicate that the RiPPs may be accommodated in the large $\mathrm{McjD}$ cavity in such a way to prevent efficient ATP hydrolysis or interfere with NBD dimerization and subsequent reduction of ATP hydrolysis.

The Natural Product ABC Transporter CapD Cannot Transport MccJ25. Taken together, our data show that McjD is highly specific for its substrate MccJ25. In order to obtain evidence on the general relevance of the substrate specificity within the peptide-derived natural product $A B C$ transporters, we investigated if the lasso peptide $A B C$ transporter $C a p D$, which exports capistruin and confers Burkholderia species with immunity to this antibacterial lasso peptide ${ }^{41}$ (Figure 5a), is able to export MccJ25. CapD was selected since it can be overexpressed in E. coli cells, purified, and retains its basal ATPase activity upon purification (Supporting Information Figure 8), suggesting that it is suitable to investigate its ability to transport MccJ25; we did not measure the ligand induced ATPase activity of CapD with capistruin due to low yields of produced peptide. In the presence of MccJ25, drug hypersensitive cells that overexpress $\mathrm{McjD}$ are capable of growing to high density (Figure 5b) compared to those that are either overexpressing an empty plasmid (control; Supporting Information Figure 9) or overexpressing CapD (Figure 5c), suggesting that CapD cannot confer immunity to MccJ25. Under these conditions, it is observed that MccJ25 is lethal to cells. On the other hand, the equivalent experiment where $\mathrm{McjD}$ is expressed in Burkholderia species in the presence of capistruin could not be performed since the genetic manipulation for 
a

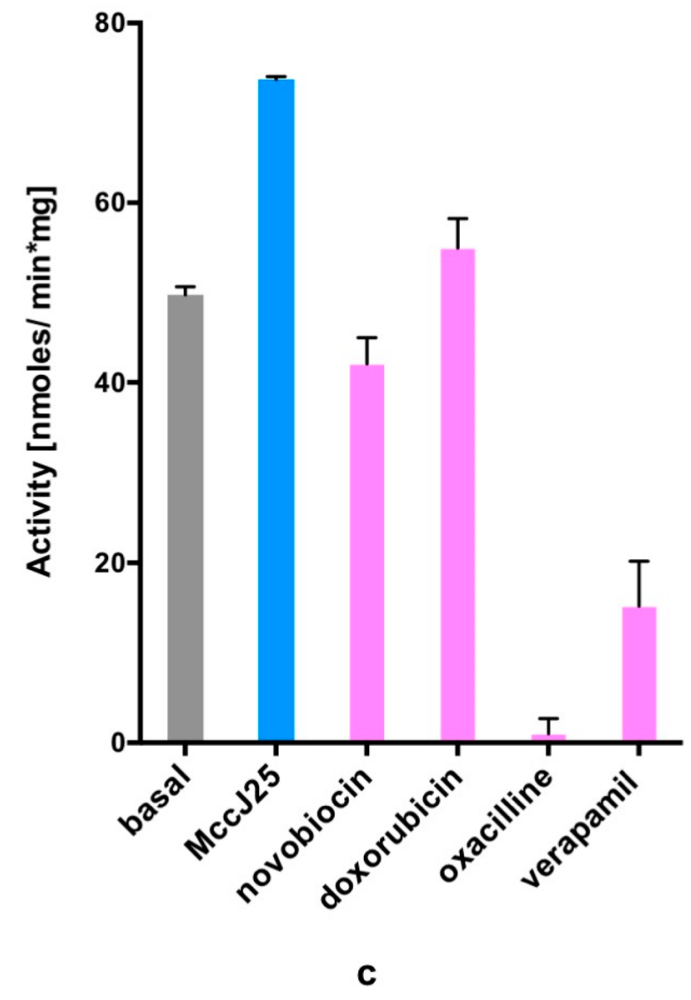

b

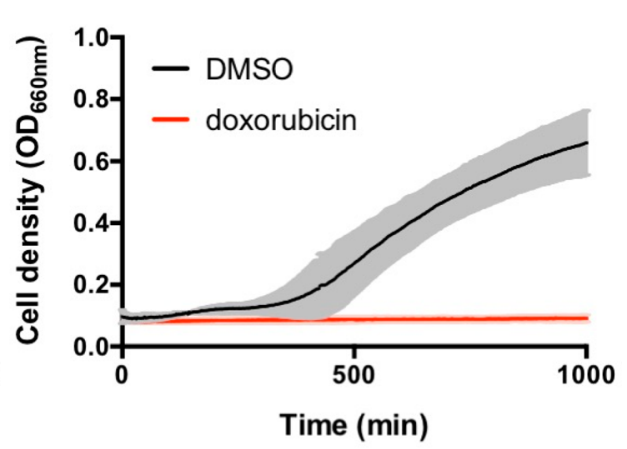

d

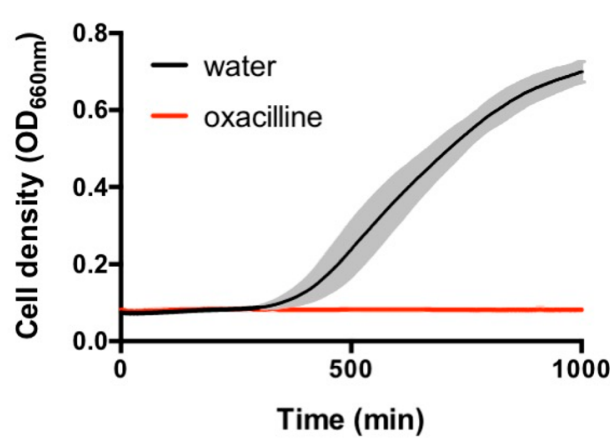

e

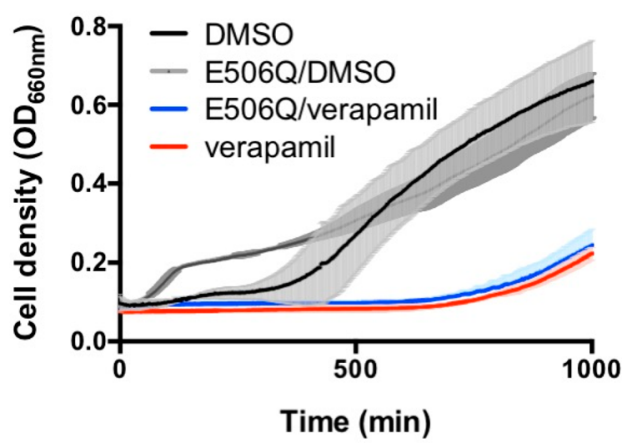

Figure 4. Functional characterization of McjD in the presence of different drugs. (a) The basal ATPase activity of McjD is induced by Mccj25 (same data used as in Figure $3 \mathrm{~b}$ for clarity) but not by other drugs. Error bars are shown for all measurements (mean \pm SEM; $n=3$ ). (b) Growth of drug hypersensitive E. coli $\Delta \mathrm{acrAB}$ expressing McjD with (red) or without (black) novobiocin $\left(5 \mu \mathrm{g} \mathrm{mL}^{-1}\right)$, (c) doxorubicin $\left(5 \mu \mathrm{g} \mathrm{mL} L^{-1}\right)$, (d) oxacillin $(5 \mu \mathrm{g}$ $\left.\mathrm{mL}^{-1}\right)$, and (e) verapamil $\left(450 \mu \mathrm{g} \mathrm{mL}^{-1}\right)$. The E506Q mutant was used to assess if the delayed cell growth in the presence of verapamil was due to McjD active transport; the E506Q growth curves are blue with and black without verapamil. All cytotoxicity assays were performed in triplicate from three different colonies. The curves represent the mean of three independent experiments.

recombinant protein overexpression in Burkholderia species is not staightforward.
McjD Specificity. Multidrug bacterial ABC transporters (exporters) are capable of recognizing and transporting a range 
a

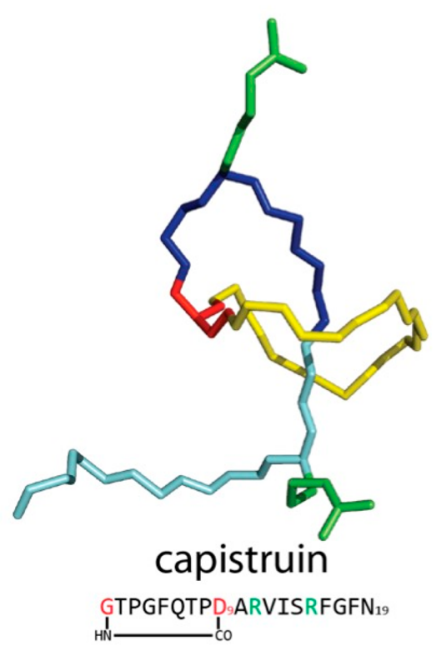

b

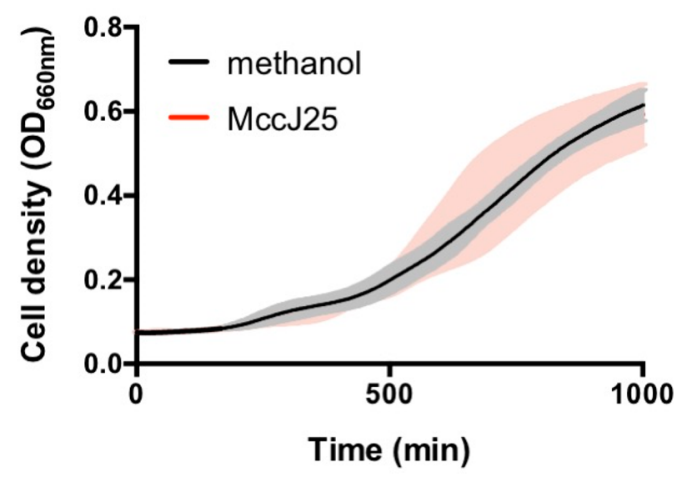

C

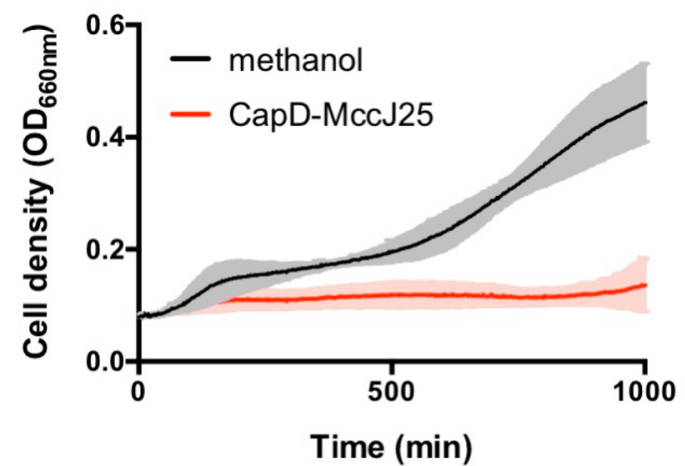

Figure 5. Functional characterization of CapD in the presence of MccJ25. (a) Structure of capistruin. Capistruin belongs to the class II lasso peptide RiPPs. Coloring as in Figure 1a. (b) Growth of drug-hypersensitive E. coli $\Delta$ acrAB expressing McjD and (c) CapD in the presence (red) or in the absence (black) of MccJ25 (10 $\mu \mathrm{M})$. In the presence of McjD, the cells grow to high density, whereas cells expressing CapD cannot confer self-immunity to MccJ25 that prevents cell growth due to the inhibition of the RNA polymerase.

of structurally and chemically unrelated compounds. These transporters are usually involved in the transport of an endogenous substrate, but somehow, they have acquired features that allow them to also transport toxic compounds such as antibiotics. Many of these antibiotics are transported by several other $\mathrm{ABC}$ transporters among different species even though there is no consensus binding site or sequence conservation within their TMDs. The TMD of ABC transporters dictates the substrate recognition since it is the first step of the transport mechanism. In this study, we have established that the ABC transporter $\mathrm{McjD}$ can only recognize and transport its substrate MccJ25 that distinguishes it from other multidrug $A B C$ transporters that has significant implications on understanding natural product biosynthesis and in particular post-translationally modified peptides.

The structures of several bacterial $A B C$ transporters have been determined but in most cases in the absence of a bound substrate to explain their mode of ligand recognition. ${ }^{17}$ The absence of $\mathrm{ABC}$ transporter-substrate structures can be attributed to either low affinity of the substrate for the transporter under crystallization conditions or absence of information about the substrate (not the case with McjD). We have previously shown that $\mathrm{McjD}$ can bind $\mathrm{Mcc} 25$ with low affinity $(100 \mu \mathrm{M})^{14}$ and that the interaction between $\mathrm{McjD}$ and $\mathrm{Mccj} 25$ can be preserved in the gas phase, ${ }^{16}$ suggesting that the complex is stable enough to be further characterized. Thus, we employed biomolecular NMR to study the interaction of McjD with MccJ25 using chemical exchange saturation transfer. Of the 21-amino-acid mature MccJ25, four residues, 8-11 (EYFV), were shown to be the primary interaction site with McjD. Deletion of the F10-V11 residues from this site of the MccJ25 lasso topology significantly reduced its apparent affinity for $\mathrm{McjD}$, as determined by nondenaturing mass spectrometry. Our nondenaturing MS data suggest that McjD has higher affinity for MccJ25 than we previously reported. ${ }^{14}$ This could be due to the McjD-GFP construct that we used in our previous study, or to the lower sensitivity of the microscale thermophoresis technique compared to native MS. These data were further validated by the absence of ligand stimulated ATPase activity of McjD in the presence of the deletion variant. Interestingly, there is no correlation between the MccJ25 residues involved in the binding with the $A B C$ transporter $\mathrm{McjD}$ and with the iron siderophore receptor FhuA. ${ }^{13}$ In the case of the McjD-MccJ25 interaction, all the interacting residues are found in the loop region, whereas in the case of FhuA they are found in both the loop and macrolactam ring. In our previous study, we identified F86, N134, and N302 as a group of residues in the McjD cavity that are important for the recognition of MccJ25. ${ }^{14,42}$ In our proposed model of McjDMccJ25 interaction, ${ }^{14} \mathrm{McjD}^{\mathrm{V} 83}$ was forming a van der Waals interaction with $\mathrm{F} 10$ and $\mathrm{V} 11$ of $\mathrm{MccJ} 25, \mathrm{McjD}^{\mathrm{F} 86} \mathrm{t}$-shaped stacked with F10 and a van der Waals interaction with the backbone of $\mathrm{E} 8$ and $\mathrm{Mcj} \mathrm{D}^{\mathrm{R} 141}$ hydrogen-bonded with the backbone of I17 and side chain of S18. Moreover, the model revealed that with this mode of binding, weaker interactions by residues S18 and I17 of MccJ25 are established with the ABC transporter, an observation that may explain the secondary region with intermediate CEST saturation. These observations 
provide further evidence of the possible binding pose of MccJ25 in the McjD cavity.

$\mathrm{ABC}$ transporters contain a large binding cavity that has been linked with multidrug resistance since they can accommodate a variety of structurally and chemically unrelated compounds. The McjD cavity is very well-defined, and it is around $5900 \AA^{3}$, which could enable it to bind different RiPPs or drugs. Using ligand induced ATPase assays, we screened different classes of RiPPs, including representatives of the three classes of lasso peptide and the flexible lantipeptide, nisin. None of these natural products was capable of stimulating the ATPase activity of McjD in proteoliposomes, even though some of these peptides have a similar topology to MccJ25, suggesting that they are possibly not transported. Since these peptides do not have antibacterial activity against $E$. coli, we could not perform cytotoxicity assays to evaluate the possibility of slow transport by McjD. If they were indeed slowly transported in vitro (inside the proteoliposomes), we would have observed a significant drop in the ATPase activity of $\mathrm{McjD}$. This is the first evidence that $\mathrm{ABC}$ transporters found at the end of the biosynthetic cluster of RiPPs are very specific for their endogenous toxic peptide. In addition, we investigated if $\mathrm{Mcj} \mathrm{D}$ could confer multidrug resistance to different antibiotics and cancer drugs. Both our ligand-induced ATPase and cytotoxicity data suggest that $\mathrm{McjD}$ cannot provide protection to a drug hypersensitive E. coli cell line in the presence of structurally and chemically different drugs. We have previously shown that the dye Hoechst can induce the ATPase activity of $\mathrm{McjD}$ around 2-fold, and it can be transported inside proteoliposomes weakly, suggesting that it is possibly interacting nonspecifically with $\mathrm{McjD}$ since it can be outcompeted by MccJ25. ${ }^{15}$ Finally, we showed that the lasso peptide capistruin $\mathrm{ABC}$ transporter CapD from Burkholderia cannot provide selfimmunity to MccJ25 in a drug hypersensitive E. coli cell line, further suggesting that these $\mathrm{ABC}$ transporters have evolved to provide cells with self-immunity against toxic peptides that the cells produce.

In conclusion, we have shown that the $\mathrm{ABC}$ transporter $\mathrm{Mcj} \mathrm{D}$ can only recognize and transport its actual substrate MccJ25, which distinguishes it from other multidrug $\mathrm{ABC}$ transporters. We propose that specific interactions between $\mathrm{McjD}$ and MccJ25 provide the transporter with a high degree of specificity, whereas multidrug $\mathrm{ABC}$ transporter promiscuity is due to nonspecific interactions. In most cases, these transporters are regulated under the same operon, and their upregulation is only initiated when the biosynthesis of the peptides occurs; therefore, they are not under pressure to recognize and transport a wide variety of substrates such as other multidrug exporters. The uncharacterized E. coli $\mathrm{ABC}$ transporter YojI is known to confer resistance to $\mathrm{MccJ} 25{ }^{43}$ thus suggesting that bacterial cells have evolved resistance to natural products (it is currently unknown if YojI can confer multidrug resistance). Our results shed some light on the evolution of $\mathrm{ABC}$ transporters to confer multidrug resistance in bacteria. These findings also have tremendous importance in understanding biosynthetic clusters as well as in using bacterial cell factories, synthetic biology, for the generation of novel bioactive compounds. In this context, our investigation provides a comprehensive characterization of the final step of the maturation process of these natural products, by revealing a fundamental mechanism of recognition by membrane bound $\mathrm{ABC}$ transporters.

\section{METHODS}

McjD Overexpression, Purification, and Reconstitution in Bicelles. McjD was overexpressed, purified in $0.03 \%$ dodecylmaltopyranoside (DDM), and reconsituted in bicelles as before without any modifications. 14,15

CapD Cloning, Overexpression, Purification. CapD was cloned in a pET28b vector engineered with a C-terminal His6-tag and a TEV cleavage site. Expression cultures in C43 (DE3) cells were performed using Terrific Broth supplemented with $0.5 \%$ glucose. Upon reaching a 0.7 optical density at $600 \mathrm{~nm}\left(\mathrm{OD}_{600}\right)$, the cultures were cooled to $20^{\circ} \mathrm{C}$ and induced by the addition of isopropyl-beta-D-thiogalactopyranoside (IPTG) at a final concentration of $0.2 \mathrm{mM}$. Protein purification was performed as for McjD. ${ }^{14,15}$ In brief, after solubilization from the membranes using $1 \% \mathrm{DDM}$, the protein was purified by affinity chromatography using Ni-NTA resin, followed by TEV cleavage in dialysis buffer containing 0.03\% DDM. The protein sample was further purified by size exclusion chromatography (SEC) before biochemical analyses (Supporting Information Figure 8).

MccJ25, MccJ25- $\Delta F$, and ${ }^{15} \mathrm{~N}-$ MccJ25 Purification. MccJ25 and ${ }^{15} \mathrm{~N}$-Mccj25 labeled peptide were produced from cultures of $E$. coli K12 MC4100 harboring the plasmid pTUC202 and purified as previously described. ${ }^{44}$ The labeling efficiency was determined by mass spectrometry (Supporting Information Figure 10). The lasso topology of MccJ25, ${ }^{15} \mathrm{~N}-\mathrm{MccJ} 25$, and MccJ25- $\Delta \mathrm{FV}$ was also verified by mass spectrometry.

Lasso Peptides and Lantipeptide Used for ATPase Assays. Production, purification, and characterization of the type I (siamycin I), type II (burhizin/caulosegnin I/sphingopyxin I/sphingonodin I/ xanthomonin I), and type III (BI-32169) lasso peptides that were used for the ATPase assays have been described previously. ${ }^{36,37,38,39}$ Nisin from Lactococcus lactis was purchased from Sigma (N5764).

Assignment of the NMR Resonances of MccJ25. Resonances of MccJ25 were assigned using a ${ }^{13} \mathrm{C}-{ }^{15} \mathrm{~N}$ labeled peptide dissolved at a concentration of $150 \mu \mathrm{M}$ in $20 \mathrm{mM}$ phosphate buffer at $\mathrm{pH} 7.5$ containing $150 \mathrm{mM}$ of $\mathrm{NaCl}$. All NMR spectra in this study were recorded at $310 \mathrm{~K}$ using an 800 or $950 \mathrm{MHz}$ instrument equipped with cryoprobe TCI. Assignment of the resonances in the ${ }^{1} \mathrm{H}-{ }^{15} \mathrm{~N}$ HSQC was obtained via a combination of 3D spectra (CBCAcoNH, HNCACB, $\mathrm{HNCO}, \mathrm{HNcaCO}$ ) to reconstruct backbone connectivities. A $3 \mathrm{D}{ }^{15} \mathrm{~N}$ edited NOESY-HSQC spectrum was recorded at $310 \mathrm{~K}$ to assist resonance assignments. The assignment of the minor form was facilitated by $\mathrm{ZZ}$ exchange ${ }^{15} \mathrm{~N}-\mathrm{HSQC}$ based spectroscopy (mixing time $1 \mathrm{~s})$ at high temperature $(318 \mathrm{~K})$ to speed up the exchange process.

Chemical Exchange Saturation Transfer. CEST measurements were based on ${ }^{1} \mathrm{H}-{ }^{15} \mathrm{~N}$ HSQC experiments by applying constant wave saturation of $500 \mathrm{~Hz}$ in the ${ }^{15} \mathrm{~N}$ channel. As the exchange is probed between unbound MccJ25 (having sharp resonances) and the slow tumbling $250 \mathrm{kDa}$ complex with $\mathrm{McjD}$ embedded in bicelles (having significantly broad resonances), a series of large offsets was employed $(-10,-8,-6,-4,-3,-2,-1.5,0,1.5,2,3,4,6,8$, and $10 \mathrm{kHz})$, resulting in CEST profiles of symmetric shape (Supporting Information Figure 1). An additional spectrum, saturated at $-100 \mathrm{kHz}$, was recorded as a reference. The ${ }^{1} \mathrm{H}-{ }^{15} \mathrm{~N}-\mathrm{HSQC}$ spectra recorded using different offsets were made with a data matrix consisting of $2048\left(t_{2},{ }^{1} \mathrm{H}\right) \times 200$ $\left(t_{1},{ }^{15} \mathrm{~N}\right)$ complex points. CEST were measured using a concentration of $100 \mu \mathrm{M}$ of MccJ25 and $8 \mu \mathrm{M}$ of McjD reconstituted in bicelles. A saturation index was calculated for each residue of MccJ25 as follows:

$$
\text { saturation index }=\sum_{j=-3}^{j=3}\left(1-I_{j} / I_{0}\right)
$$

where $j$ is the offset employed in each CEST measurement and $I_{j}$ and $I_{0}$ are the intensities of the peak corresponding to the amide of the residue in the spectra recorded with the offset $j$ and with an offset at $-100 \mathrm{kHz}$ (reference spectrum). The saturation indexes were calculated using only the CEST measurements made with offsets ranging from $-3 \mathrm{kHz}$ to +3 $\mathrm{kHz}$ (excluding $0 \mathrm{kHz}$ ), which are significantly affected by the binding with the $\mathrm{ABC}$ transporter.

Structural Refinement Using Chemical Shift Restrained MD Simulations. Chemical shifts of the two conformations of MccJ25 were 
employed in restrained molecular dynamics simulations using the CamShift method, ${ }^{45}$ following established protocols that have been highly successful in characterizing the structures of small peptides using the information contained in NMR chemical shifts. ${ }^{20,46}$

The restrained molecular dynamics simulations were performed by averaging chemical shift restraints over four replicas, as previously described, ${ }^{20,46}$ using the structure of MccJ25 with PDB code 1Q71. ${ }^{7}$

The calculations were made using an implementation of the GROMACS package ${ }^{47}$ that allows the simulations to be restrained using the CamShift program. ${ }^{45}$ Each of the four replicas was equilibrated separately by starting from independent equilibrations of the starting structure of MccJ25 accommodated in a dodecahedron box of $121 \mathrm{~nm}^{3}$ in volume. The box was filled with 3869 explicit waters and energy minimized. The simulations were carried out using the CHARMM27 force field and the TIP $3 p$ water model.

A time step of $2 \mathrm{fs}$ was used together with LINCS constraints. The van der Waals and electrostatic interactions were cut off at $0.9 \mathrm{~nm}$, and longrange electrostatic effects were treated with the particle mesh Ewald method. All the simulations were carried out in the canonical ensemble by keeping the volume fixed and by setting the system temperature with the V-rescale thermostat.

The replica-averaged restrained molecular dynamics simulations were carried out using the following protocol. The four replicas evolved through a series of annealing cycles between 310 and $380 \mathrm{~K}$, each cycle being composed of $100 \mathrm{ps}$ of simulation at $310 \mathrm{~K}$ followed by $100 \mathrm{ps}$ of simulation in which the temperature of the system was increased up to $380 \mathrm{~K}$ and $100 \mathrm{ps}$ of simulation carried at a constant temperature of 380 $\mathrm{K}$. The final part of the cycle allowed the system to cool slowly from 380 to $310 \mathrm{~K}$ in a step of $300 \mathrm{ps}$. During these cycles, the experimental restraints were imposed as averages over the four replicas. The total amount of sampling in each system simulated was $1 \mu \mathrm{s}(250.2 \mathrm{~ns}$ per replica equivalent to 417 cycles). Primary and secondary conformations of MccJ25 were refined using two independent runs.

Nondenaturing Mass Spectrometry Analysis. DDM solubilized McjD was buffer exchanged to $200 \mathrm{mM}$ ammonium acetate supplemented with $0.16 \%$ OGNG detergent as previously described. ${ }^{16}$ Nondenaturing mass spectra were acquired on a modified Q-TOF mass spectrometer for high $\mathrm{m} / z$ transmission and for higher activation conditions. The mass spectrometer was set under the same conditions as before. ${ }^{16}$ Samples were introduced into the ion source via in-house prepared gold-coated capillaries under static nanospray. MccJ25 was mixed with $\mathrm{McjD}$ protein solution at different concentrations (Supporting Information Figure 7). MccJ25- $\Delta \mathrm{FV}$ was added at a final concertation of $8 \mu \mathrm{M}$. The data for the apo-McjD and in complex with MccJ25 in Figure 3C have previously been published, ${ }^{16}$ and they have been reproduced in this manuscript to show the difference with the MccJ25- $\Delta$ FV variant.

ATPase Activity of Proteoliposome Reconstituted McjD. $\mathrm{McjD}$ was reconstituted in proteoliposomes using the rapid dilution protocol as in ref 15 . The ATPase activity of $\mathrm{McjD}$ in the presence of different peptides and antibiotics was measured as previously described. $^{14,15}$ The final concentration of the peptides in the assay was $1 \mathrm{mM}$ made in $100 \%$ dimethyl sulfoxide (DMSO). Antibiotics and anticancer drugs were also added at $1 \mathrm{mM}$ final concentration made either in water or in $100 \%$ DMSO depending on the compound solubility. The final DMSO concentration was $1 \%(\mathrm{v} / \mathrm{v})$ for all the compounds tested.

Cytotoxic Assays in Drug Hypersensitive Cells. Overnight cultures of E. coli $\triangle \mathrm{acrAB}$, harboring McjD-pET28b, McjD-E506QpET28b, CapD-pET28b, or empty pET28b plasmid, were inoculated into an LB medium containing $50 \mu \mathrm{g} \mathrm{mL}^{-1}$ kanamycin. At an optical density at $600 \mathrm{~nm}(\mathrm{OD} 600)$ of 0.8 , cells were diluted to an $\mathrm{OD}_{600}$ of 0.06 in fresh medium containing $0.07 \mathrm{mM}$ IPTG in a 96-well plate to which the $\operatorname{MccJ} 25(10 \mu \mathrm{M})$, the antibiotics, $\left(5 \mu \mathrm{g} \mathrm{mL}^{-1}\right)$, and verapamil $(450$ $\mu \mathrm{g} \mathrm{mL}^{-1}$ ) were added. Growth was followed over time at $\mathrm{OD}_{600}$ at $37^{\circ} \mathrm{C}$ in a Molecular Devices SpectraMax M2Multimode Microplate Reader (Molecular Devices). The same experimental procedures were followed for the control experiments in which the same volume of water, DMSO, or methanol was used.
Each growth experiment was performed from three independent biological clones, and each measurement was performed in triplicate.

\section{ASSOCIATED CONTENT}

\section{S Supporting Information}

The Supporting Information is available free of charge on the ACS Publications website at DOI: 10.1021/acschembio.8b00226.

MccJ25 NMR spectra and saturation profiles, nondenaturing mass spectra titration, purification and characterization of the $\mathrm{ABC}$ transporter CapD (PDF)

\section{AUTHOR INFORMATION}

\section{Corresponding Author}

*E-mail: kbeis@imperial.ac.uk.

ORCID

Konstantinos Beis: 0000-0001-5727-4721

Present Address

Current address

\section{Author Contributions}

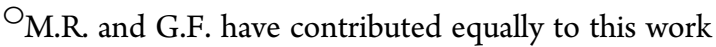

\section{Author Contributions}

K.B. designed and managed the overall project. M.R. and H.G.C. purified proteins for NMR analysis. G.F. and E.L. measured the NMR data. G.F., E.L., and A.D.S. analyzed the NMR data. M.R. performed ATPase and cytotoxicity assays. S.Z. and S.R. produced and purified the MccJ25. J.D.H. and M.A.M. produced and purified the class I-III peptides used in the ATPase assays. S.M. and C.V.R performed and analyzed the mass spectrometry data. K.B. wrote the manuscript with help from the other authors.

Notes

The authors declare no competing financial interest.

\section{ACKNOWLEDGMENTS}

We would like to thank $H$. van Ween for providing us with the drug hypersensitive strain. J.D.H. is supported by a Deutsche Forschungsgemeinschaft (DFG) Research Fellowship (grant number HE 7699/1-1). This work was supported by the Leverhulme Trust (RPG-2015-350 to A.D.S.) and the Medical Research Council (MR/N020103/1 to K.B. and A.D.S.).

\section{REFERENCES}

(1) Newman, D. J., and Cragg, G. M. (2016) Natural Products as Sources of New Drugs from 1981 to 2014. J. Nat. Prod. 79, 629-661.

(2) Dang, T., and Sussmuth, R. D. (2017) Bioactive Peptide Natural Products as Lead Structures for Medicinal Use. Acc. Chem. Res. 50, $1566-1576$.

(3) Strieker, M., Tanovic, A., and Marahiel, M. A. (2010) Nonribosomal peptide synthetases: structures and dynamics. Curr. Opin. Struct. Biol. 20, 234-240.

(4) Ortega, M. A., and van der Donk, W. A. (2016) New Insights into the Biosynthetic Logic of Ribosomally Synthesized and Post-translationally Modified Peptide Natural Products. Cell Chem. Biol. 23, 31-44.

(5) Arnison, P. G., Bibb, M. J., Bierbaum, G., Bowers, A. A., Bugni, T. S., Bulaj, G., Camarero, J. A., Campopiano, D. J., Challis, G. L., Clardy, J., Cotter, P. D., Craik, D. J., Dawson, M., Dittmann, E., Donadio, S., Dorrestein, P. C., Entian, K. D., Fischbach, M. A., Garavelli, J. S., Goransson, U., Gruber, C. W., Haft, D. H., Hemscheidt, T. K., Hertweck, C., Hill, C., Horswill, A. R., Jaspars, M., Kelly, W. L., Klinman, J. P., Kuipers, O. P., Link, A. J., Liu, W., Marahiel, M. A., Mitchell, D. A., Moll, G. N., Moore, B. S., Muller, R., Nair, S. K., Nes, I. F., Norris, G. E., Olivera, B. M., Onaka, H., Patchett, M. L., Piel, J., Reaney, M. J., Rebuffat, S., Ross, R. P., Sahl, H. G., Schmidt, E. W., Selsted, M. E., Severinov, K., 
Shen, B., Sivonen, K., Smith, L., Stein, T., Sussmuth, R. D., Tagg, J. R., Tang, G. L., Truman, A. W., Vederas, J. C., Walsh, C. T., Walton, J. D., Wenzel, S. C., Willey, J. M., and van der Donk, W. A. (2013) Ribosomally synthesized and post-translationally modified peptide natural products: overview and recommendations for a universal nomenclature. Nat. Prod. Rep. 30, 108-160.

(6) Funk, M. A., and van der Donk, W. A. (2017) Ribosomal Natural Products, Tailored To Fit. Acc. Chem. Res. 50, 1577-1586.

(7) Rosengren, K. J., Clark, R. J., Daly, N. L., Goransson, U., Jones, A., and Craik, D. J. (2003) Microcin J25 has a threaded sidechain-tobackbone ring structure and not a head-to-tail cyclized backbone. J. Am. Chem. Soc. 125, 12464-12474.

(8) Yan, K. P., Li, Y., Zirah, S., Goulard, C., Knappe, T. A., Marahiel, M. A., and Rebuffat, S. (2012) Dissecting the maturation steps of the lasso peptide microcin J25 in vitro. ChemBioChem 13, 1046-1052.

(9) Adelman, K., Yuzenkova, J., La Porta, A., Zenkin, N., Lee, J., Lis, J. T., Borukhov, S., Wang, M. D., and Severinov, K. (2004) Molecular mechanism of transcription inhibition by peptide antibiotic Microcin J25. Mol. Cell 14, 753-762.

(10) Rebuffat, S. (2012) Microcins in action: amazing defence strategies of Enterobacteria. Biochem. Soc. Trans. 40, 1456-1462.

(11) de Cristobal, R. E., Solbiati, J. O., Zenoff, A. M., Vincent, P. A., Salomon, R. A., Yuzenkova, J., Severinov, K., and Farias, R. N. (2006) Microcin J25 uptake: His5 of the MccJ25 lariat ring is involved in interaction with the inner membrane MccJ25 transporter protein SbmA. J. Bacteriol. 188, 3324-3328.

(12) Runti, G., Del Carmen Lopez Ruiz, M., Stoilova, T., Hussain, R., Jennions, M., Choudhury, H. G., Benincasa, M., Gennaro, R., Beis, K., and Scocchi, M. (2013) Functional characterization of SbmA, a bacterial inner membrane transporter required for importing the antimicrobial peptide Bac7(1-35). J. Bacteriol. 195, 5343-5351.

(13) Mathavan, I., Zirah, S., Mehmood, S., Choudhury, H. G., Goulard, C., Li, Y., Robinson, C. V., Rebuffat, S., and Beis, K. (2014) Structural basis for hijacking siderophore receptors by antimicrobial lasso peptides. Nat. Chem. Biol. 10, 340-342.

(14) Choudhury, H. G., Tong, Z., Mathavan, I., Li, Y., Iwata, S., Zirah, S., Rebuffat, S., van Veen, H. W., and Beis, K. (2014) Structure of an antibacterial peptide ATP-binding cassette transporter in a novel outward occluded state. Proc. Natl. Acad. Sci. U. S. A. 111, 9145-9150.

(15) Bountra, K., Hagelueken, G., Choudhury, H. G., Corradi, V., El Omari, K., Wagner, A., Mathavan, I., Zirah, S., Yuan Wahlgren, W., Tieleman, D. P., Schiemann, O., Rebuffat, S., and Beis, K. (2017) Structural basis for antibacterial peptide self-immunity by the bacterial ABC transporter McjD. EMBO J. 36, 3062.

(16) Mehmood, S., Corradi, V., Choudhury, H. G., Hussain, R., Becker, P., Axford, D., Zirah, S., Rebuffat, S., Tieleman, D. P., Robinson, C. V., and Beis, K. (2016) Structural and Functional Basis for Lipid Synergy on the Activity of the Antibacterial Peptide ABC Transporter McjD. J. Biol. Chem. 291, 21656-21668.

(17) Beis, K. (2015) Structural basis for the mechanism of $A B C$ transporters. Biochem. Soc. Trans. 43, 889-893.

(18) Lee, J. Y., Yang, J. G., Zhitnitsky, D., Lewinson, O., and Rees, D. C. (2014) Structural basis for heavy metal detoxification by an Atm1-type ABC exporter. Science 343, 1133-1136.

(19) Mi, W., Li, Y., Yoon, S. H., Ernst, R. K., Walz, T., and Liao, M. (2017) Structural basis of MsbA-mediated lipopolysaccharide transport. Nature 549, 233-237.

(20) Krieger, J. M., Fusco, G., Lewitzky, M., Simister, P. C., Marchant, J., Camilloni, C., Feller, S. M., and De Simone, A. (2014) Conformational recognition of an intrinsically disordered protein. Biophys. J. 106, 1771-1779.

(21) Milojevic, J., Esposito, V., Das, R., and Melacini, G. (2007) Understanding the molecular basis for the inhibition of the Alzheimer's Abeta-peptide oligomerization by human serum albumin using saturation transfer difference and off-resonance relaxation NMR spectroscopy. J. Am. Chem. Soc. 129, 4282-4290.

(22) Huang, H., Milojevic, J., and Melacini, G. (2008) Analysis and optimization of saturation transfer difference NMR experiments designed to map early self-association events in amyloidogenic peptides. J. Phys. Chem. B 112, 5795-5802.

(23) Fawzi, N. L., Ying, J., Ghirlando, R., Torchia, D. A., and Clore, G. M. (2011) Atomic-resolution dynamics on the surface of amyloid-beta protofibrils probed by solution NMR. Nature 480, 268-272.

(24) Vallurupalli, P., Bouvignies, G., and Kay, L. E. (2012) Studying "invisible" excited protein states in slow exchange with a major state conformation. J. Am. Chem. Soc. 134, 8148-8161.

(25) Fusco, G., Pape, T., Stephens, A. D., Mahou, P., Costa, A. R., Kaminski, C. F., Kaminski Schierle, G. S., Vendruscolo, M., Veglia, G., Dobson, C. M., and De Simone, A. (2016) Structural basis of synaptic vesicle assembly promoted by alpha-synuclein. Nat. Commun. 7, 12563.

(26) Algamal, M., Ahmed, R., Jafari, N., Ahsan, B., Ortega, J., and Melacini, G. (2017) Atomic-resolution map of the interactions between an amyloid inhibitor protein and amyloid beta (Abeta) peptides in the monomer and protofibril states. J. Biol. Chem. 292, 17158-17168.

(27) Ahmed, R., VanSchouwen, B., Jafari, N., Ni, X., Ortega, J., and Melacini, G. (2017) Molecular Mechanism for the (-)-Epigallocatechin Gallate-Induced Toxic to Nontoxic Remodeling of Abeta Oligomers. J. Am. Chem. Soc. 139, 13720-13734.

(28) Ducasse, R., Yan, K. P., Goulard, C., Blond, A., Li, Y., Lescop, E., Guittet, E., Rebuffat, S., and Zirah, S. (2012) Sequence determinants governing the topology and biological activity of a lasso peptide, microcin J25. ChemBioChem 13, 371-380.

(29) Pavlova, O., Mukhopadhyay, J., Sineva, E., Ebright, R. H., and Severinov, K. (2008) Systematic structure-activity analysis of microcin J25. J. Biol. Chem. 283, 25589-25595.

(30) Mehmood, S., Allison, T. M., and Robinson, C. V. (2015) Mass spectrometry of protein complexes: from origins to applications. Annu. Rev. Phys. Chem. 66, 453-474.

(31) Kimura, Y., Kioka, N., Kato, H., Matsuo, M., and Ueda, K. (2007) Modulation of drug-stimulated ATPase activity of human MDR1/Pglycoprotein by cholesterol. Biochem. J. 401, 597-605.

(32) Eckford, P. D., and Sharom, F. J. (2008) Functional characterization of Escherichia coli MsbA: interaction with nucleotides and substrates. J. Biol. Chem. 283, 12840-12850.

(33) Maksimov, M. O., and Link, A. J. (2014) Prospecting genomes for lasso peptides. J. Ind. Microbiol. Biotechnol. 41, 333-344.

(34) Zhao, N., Pan, Y., Cheng, Z., and Liu, H. (2016) Lasso peptide, a highly stable structure and designable multifunctional backbone. Amino Acids 48, 1347-1356.

(35) Li, Y., Zirah, S., and Rebuffat, S. (2015) Lasso peptides: Bacterial strategies to make and maintain bioactive entangled scaffolds, Springer, New York.

(36) Hegemann, J. D., Zimmermann, M., Zhu, S., Klug, D., and Marahiel, M. A. (2013) Lasso peptides from proteobacteria: Genome mining employing heterologous expression and mass spectrometry. Biopolymers 100, 527-542.

(37) Hegemann, J. D., Zimmermann, M., Xie, X., and Marahiel, M. A. (2013) Caulosegnins I-III: a highly diverse group of lasso peptides derived from a single biosynthetic gene cluster. J. Am. Chem. Soc. 135, 210-222.

(38) Fage, C. D., Hegemann, J. D., Nebel, A. J., Steinbach, R. M., Zhu, S., Linne, U., Harms, K., Bange, G., and Marahiel, M. A. (2016) Structure and Mechanism of the Sphingopyxin I Lasso Peptide Isopeptidase. Angew. Chem., Int. Ed. 55, 12717-12721.

(39) Hegemann, J. D., Zimmermann, M., Zhu, S., Steuber, H., Harms, K., Xie, X., and Marahiel, M. A. (2014) Xanthomonins I-III: a new class of lasso peptides with a seven-residue macrolactam ring. Angew. Chem., Int. Ed. 53, 2230-2234.

(40) Egan, K., Field, D., Rea, M. C., Ross, R. P., Hill, C., and Cotter, P. D. (2016) Bacteriocins: Novel Solutions to Age Old Spore-Related Problems? Front. Microbiol. 7, 461.

(41) Knappe, T. A., Linne, U., Zirah, S., Rebuffat, S., Xie, X., and Marahiel, M. A. (2008) Isolation and structural characterization of capistruin, a lasso peptide predicted from the genome sequence of Burkholderia thailandensis E264. J. Am. Chem. Soc. 130, 11446-11454.

(42) Gu, R. X., Corradi, V., Singh, G., Choudhury, H. G., Beis, K., and Tieleman, D. P. (2015) Conformational Changes of the Antibacterial 
Peptide ATP Binding Cassette Transporter McjD Revealed by Molecular Dynamics Simulations. Biochemistry 54, 5989-5998.

(43) Delgado, M. A., Vincent, P. A., Farias, R. N., and Salomon, R. A. (2005) YojI of Escherichia coli functions as a microcin J25 efflux pump. J. Bacteriol. 187, 3465-3470.

(44) Zirah, S., Afonso, C., Linne, U., Knappe, T. A., Marahiel, M. A., Rebuffat, S., and Tabet, J. C. (2011) Topoisomer differentiation of molecular knots by FTICR MS: lessons from class II lasso peptides. J. Am. Soc. Mass Spectrom. 22, 467-479.

(45) Kohlhoff, K. J., Robustelli, P., Cavalli, A., Salvatella, X., and Vendruscolo, M. (2009) Fast and accurate predictions of protein NMR chemical shifts from interatomic distances. J. Am. Chem. Soc. 131, 13894-13895.

(46) Fusco, G., De Simone, A., Arosio, P., Vendruscolo, M., Veglia, G., and Dobson, C. M. (2016) Structural Ensembles of Membrane-bound alpha-Synuclein Reveal the Molecular Determinants of Synaptic Vesicle Affinity. Sci. Rep. 6, 27125.

(47) Hess, B., Kutzner, C., van der Spoel, D., and Lindahl, E. (2008) GROMACS 4: Algorithms for highly efficient, load-balanced, and scalable molecular simulation. J. Chem. Theory Comput. 4, 435-447. 